\title{
Evolução diferencial com mutação ordenada em problemas de otimização monobjetivo com restrições de caixa
}

\author{
Dênis E. C. Vargas ${ }^{1}$ \\ DM/CEFET-MG, Belo Horizonte, MG \\ Rafael de Paula Garcia ${ }^{2}$ \\ DAU/UFV, Viçosa, MG \\ Afonso C. C. Lemonge ${ }^{3}$ \\ MAC/UFJF, Juiz de Fora, MG
}

\begin{abstract}
Resumo. A Evolução Diferencial (ED) está entre os algoritmos evolucionistas mais eficientes para lidar com problemas de otimização. Sua proposição original utiliza o esquema clássico DE/Rand para selecionar aleatoriamente soluções candidatas da população para o processo de mutação, sem considerar qualquer ordenação entre elas. Recententemente foi proposto o esquema DE/Order para problemas multi-objetivo, uma estratégia de ordenação entre as soluções selecionadas para a mutação. O algoritmo com o esquema DE/Order apresentou melhores resultados em problemas de otimização multi-objetivo quando comparado ao DE/Rand. Esse trabalho avalia a estratégia de mutação DE/Order em problemas de otimização monobjetivo com restrições de caixa. A performance desta estratégia foi comparada com duas outras já consolidadas na literatura, DE/Rand e DE/Best, ao serem aplicadas a problemas monobjetivos benchmark da competição do IEEE Congress on Evolutionary Computation - CEC 2021. Os resultados mostraram que o esquema DE/Best apresenta o pior desempenho, sugerindo convergência prematura para ótimos locais. Além disso, este trabalho mostra através de testes não paramétricos que as estratégias DE/Order e DE/Rand não demonstraram diferenças estatísticas. Concluiu-se que o DE/Order se mostra competitivo neste conjunto de problemas, apresentando-se como uma estratégia que se beneficia dos conceitos das outras duas abordagens, randomização e elitismo, porém sem ser prejudicado pela estagnação em ótimos locais.
\end{abstract}

Palavras-chave. Evolução diferencial, mutação ordenada, otimização monobjetivo.

\section{Introdução}

A Evolução Diferencial (ED) [1] é um algoritmo evolucionista que se destaca devido à sua simplicidade, aos poucos parâmetros de controle utilizados e pelo bom desempenho com rápida convergência. Na sua versão original, a ED seleciona aleatoriamente soluções candidatas da população, sem considerar qualquer ordenação, para aplicar sobre elas mutação e recombinação de suas variáveis. Esta abordagem é conhecida como DE/Rand.

Uma alternativa a este esquema de seleção foi proposta recentemente na quarta versão do algoritmo Generalized Differential Evolution (GDE4) [2], que introduziu uma ordenação dessas

\footnotetext{
${ }^{1}$ denis.vargas@cefetmg.br

${ }^{2}$ rafael.pgarcia@ufv.br

3 afonso.lemonge@ufjf.edu.br
} 
soluções antes da aplicação do processo de mutação. Nesta estratégia, três soluções são selecionadas aleatoriamente como na anterior DE/Rand, porém antes da aplicação da mutação, uma ordenação destas soluções é feita como uma tentativa de tender a busca para regiões com melhores valores de função objetivo. Os autores denominaram esse esquema de DE/Order. Os experimentos em [2] foram conduzidos em problemas de otimização multi-objetivo e os resultados da comparação revelam que o algoritmo GDE4 supera o GDE3 [3], versão anterior do algoritmo Generalized Differential Evolution e que utiliza o esquema DE/Rand.

Este trabalho se propôs a realizar uma continuidade do estudo [2] para avaliar se a boa performance do DE/Order se estende a problemas de otimização monobjetivo com restrições de caixa. A ED com a estratégia DE/Order foi aplicada aos problemas monobjetivo de otimização com restrições de caixa benchmark da Competição do IEEE Congress on Evolutionary Computation - CEC 2021 [4]. Os resultados foram comparados com os obtidos pela ED usando os esquemas clássicos DE/Rand e DE/Best nos mesmos problemas mantendo os parâmetros da ED inalterados.

\section{Problemas Monobjetivo de Otimização com Restrições de Caixa}

Problemas monobjetivo de otimização com restrições de caixa podem ser definidos como:

$$
\begin{array}{cc}
\min _{\mathbf{x}} & f(\mathbf{x}) \\
\text { onde } & \mathbf{x}=\left(x_{1}, \ldots, x_{D}\right) \\
& x_{i} \in\left[l_{i}, u_{i}\right]
\end{array}
$$

onde $l_{i}$ e $u_{i}$ são, respectivamente, os limites inferior e superior de cada entrada $x_{i}$ do vetor $\mathbf{x} \in \mathbb{R}^{n}$.

Neste trabalho, 10 problemas benchmark de otimização monobjetivo com restrições de caixa da competição do IEEE Congress on Evolutionary Computation - CEC 2021 foram adotados para os experimentos computacionais, com $D=10$ e $D=20$. Estes problemas são frequentemente adotados para comparar a performance de algoritmos evolucionistas. Suas nomenclaturas e principais características podem ser observadas na Tabela 1, enquanto que uma descrição detalhada pode ser acessada em [4].

\section{Evolução Diferencial}

A Evolução Diferencial (ED) [1] é um algoritmo evolucionista que parte de uma população inicial de $N P$ soluções candidatas, geradas aleatoriamente e a evolui ao longo de gerações. A evolução ocorre pela aplicação de operadores de mutação e recombinação sobre a população de uma geração, produzindo novas soluções candidatas. O esquema de mutação proposto na versão original da ED funciona da seguinte forma: para cada uma das $N P$ soluções candidatas $\left(\mathbf{x} \in \mathbb{R}^{n}\right.$ chamado de vetor alvo), sorteia-se três soluções na população $\mathbf{x}_{r 1}, \mathbf{x}_{r 2}$ e $\mathbf{x}_{r 3} \in \mathbb{R}^{n}$, diferentes entre si e diferentes do vetor alvo. Um vetor $\mathbf{v} \in \mathbb{R}^{n}$ (chamado de vetor doador) é então calculado pela fórmula a seguir:

$$
\mathbf{v}=\mathbf{x}_{r 1}+F\left(\mathbf{x}_{r 2}-\mathbf{x}_{r 3}\right)
$$

onde $F \in \mathbb{R}$ é um parâmetro de multiplicação maior do que zero.

O vetor experimental $\mathbf{u}$ é encontrado após a aplicação do processo de cruzamento, que mistura informações apresentadas pelas soluções $\mathbf{x}$ e v. Para isso, um inteiro aleatório $j$ entre 1 e $n$ é sorteado e para cada $i=1, \ldots, n$, um número aleatório rand $_{i}$ entre 0 e 1 também é sorteado. Se $\operatorname{rand}_{i} \leq C R$ ou $i=j$, então $u_{i}=v_{i}$. Caso contrário, $u_{i}=x_{i}$. A condição $i=j$ garante a cópia de 
Tabela 1: Funções básicas envolvidas nos 10 problemas da Competição do CEC 2021 e principal característica

\begin{tabular}{|c|c|c|}
\hline Problema & Função(ões) envolvida(s) & Característica \\
\hline 1 & Bent Cigar & Unimodal \\
\hline 2 & Schwefel & Função Básica \\
\hline 3 & Lunacek bi-Rastrigin & Função Básica \\
\hline 4 & Rosenbrock plus Griewangk & Função Básica \\
\hline 5 & Schwefel, Rastrigin e Elliptic & Função Híbrida \\
\hline 6 & Schaffer, HGBat, Rosenbrock e Schwefel & Função Híbrida \\
\hline 7 & Schaffer, HGBat, Rosenbrock, Schwefel e Elliptic & Função Híbrida \\
\hline 8 & Rastrigin, Griewank e Schwefel & Composição de Funções \\
\hline 9 & Ackley, Elliptic, Griewank e Rastrigin & Composição de Funções \\
\hline 10 & Rastrigin, Happycat, Ackley, Discus e Rosenbrock & Composição de Funções \\
\hline
\end{tabular}

pelo menos uma variável do vetor doador. Por fim, utiliza-se um critério de seleção para decidir qual das duas soluções u ou x continua na população da geração seguinte. Se $f(\mathbf{u})<f(\mathbf{x})$, então $\mathbf{u}$ substitui $\mathbf{x}$ na população da geração seguinte. Do contrário, u é descartado e $\mathbf{x}$ permanece na população.

O esquema de mutação apresentado pela Equação 2 é conhecido como DE/Rand, pois os vetores são escolhidos e classificados aleatoriamente. Na literatura existem outros esquemas clássicos como o DE/Best, que substitui o $\mathbf{x}_{r 1}$ da fórmula da Equação 2 pelo $\mathbf{x}_{b}$, o melhor de toda a população.

Recentemente foi proposto em [2] um novo esquema de mutação de ED chamado DE/Order, que introduziu uma ordenação das soluções sorteadas antes da aplicação do processo de mutação. No esquema DE/Order, as 3 soluções candidatas sorteadas $\mathbf{x}_{r 1}, \mathbf{x}_{r 2}$ e $\mathbf{x}_{r 3} \in \mathbb{R}^{n}$ são classificadas em $\mathbf{x}_{b}$ (best), $\mathbf{x}_{2 b}$ (second best) e $\mathbf{x}_{w}$ (worst) de acordo com os valores de suas funções objetivos. Os experimentos em [2] foram conduzidos em problemas de otimização multi-objetivo e a classificação considerava a posição das soluções no ranqueamento por dominância de Pareto e uma medida de diversidade chamada Crowding Distance. Os autores compararam o DE/Order apenas com o DE/Rand. Nesse trabalho, além de adaptar o DE/Order para problemas de otimização monobjetivo com restrições de caixa, também utilizou-se o esquema DE/Best para comparação. Todos os esquemas de mutação utilizados nesse trabalho estão detalhados na Tabela 2.

\section{Experimentos Computacionais}

Nesse trabalho avaliou-se a performance do esquema de mutação DE/Order para problemas de otimização monobjetivo com restrições de caixa. Eles foram comparados aos esquemas clássicos DE/Rand e DE/Best nos 10 problemas monobjetivo de otimização da Competição do IEEE Congress on Evolutionary Computation - CEC 2021, com dimensões $D=10$ e $D=20$, resultando, portanto, na experimentação em 20 diferentes problemas. Os parâmetros adotados para todos os algoritmos foram os sugeridos pela própria competição, além de serem popularmente utilizados na 
Tabela 2: Esquemas de Mutação de ED utilizados nesse trabalho.

\begin{tabular}{|c|c|c|}
\hline Esquema & Fórmula & Definição \\
\hline DE/Rand & $\mathbf{v}=\mathbf{x}_{r 1}+F\left(\mathbf{x}_{r 2}-\mathbf{x}_{r 3}\right)$ & $\mathbf{x}_{r 1}, \mathbf{x}_{r 2}$ e $\mathbf{x}_{r 3}$ aleatórios \\
\hline DE/Best & $\mathbf{v}=\mathbf{x}_{b}+F\left(\mathbf{x}_{r 1}-\mathbf{x}_{r 2}\right)$ & $\begin{array}{c}\mathbf{x}_{r 1} \text { e } \mathbf{x}_{r 2} \text { aleatórios } \\
\mathbf{x}_{b}: \begin{array}{c}\text { solução com melhor valor de função } \\
\text { objetivo em toda a população }\end{array}\end{array}$ \\
\hline DE/Order & $\mathbf{v}=\mathbf{x}_{b}+F\left(\mathbf{x}_{2 b}-\mathbf{x}_{w}\right)$ & $\begin{array}{c}\mathbf{x}_{r 1}, \mathbf{x}_{r 2} \text { e } \mathbf{x}_{r 3} \text { aleatórios } \\
\mathbf{x}_{b}, \mathbf{x}_{2 b} \text { e } \mathbf{x}_{w} \text { escolhidos entre } \mathbf{x}_{r 1}, \mathbf{x}_{r 2} \text { e } \mathbf{x}_{r 3} \\
\text { de acordo com a melhor, segunda melhor e } \\
\text { pior valor de função objetivo, respectivamente }\end{array}$ \\
\hline
\end{tabular}

literatura: $N P=100, F=0.5, C R=0.9,200.000$ e 1.000.000 de avaliações da função objetivo como critério de parada para $D=10$ e $D=20$, respectivamente, e tolerância de $1 \times 10^{-8}$. Foram conduzidas 30 execuções independentes. Apesar dos problemas da competição do CEC 2021 considerarem possibilidades de parametrizações como polarização, deslocamento e rotação, nenhuma dessas configurações foram adotadas nesse trabalho.

A competição propõe que os algoritmos sejam avaliados segundo a soma de dois scores. Para o primeiro, $S_{\text {core }}$, inicialmente, cada algoritmo tem uma Soma de Erros Normalizados $(S N E)$ calculada através da fórmula

$$
S N E=\frac{1}{2} \sum_{i=1}^{20} n e_{i} \quad \text { onde } \quad n e=\frac{f\left(\mathbf{x}_{\text {best }}\right)-f\left(\mathbf{x}^{*}\right)}{f\left(\mathbf{x}_{\text {best }}\right)_{\max }-f\left(\mathbf{x}^{*}\right)}
$$

sendo $f\left(\mathbf{x}_{\text {best }}\right)$ o melhor resultado do algoritmo nas 30 execuções independentes, $f\left(\mathbf{x}^{*}\right)$ o valor ótimo conhecido da função e $f\left(\mathbf{x}_{\text {best }}\right)_{\text {max }}$ o maior $f\left(\mathbf{x}_{\text {best }}\right)$ entre todos os algoritmos para a função considerada. Uma vez que $S N E$ foi determinado para todos os algoritmos, o $S$ core $e_{1}$ é calculado como

$$
\text { Score }_{1}=\left(1-\frac{S N E-S N E_{\text {min }}}{S N E}\right) \times 50
$$

onde $S N E_{\text {min }}$ é o menor $S N E$ entre todos os algoritmos.

Para o ccore $_{2}$, considera-se para cada algoritmo uma Soma de Ranks $(S R)$ calculada através da fórmula

$$
S R=\frac{1}{2} \sum_{i=1}^{20} \operatorname{Rank}_{i}
$$

onde $\operatorname{Rank}_{i}$ é a classificação do algoritmo entre todos os algoritmos para uma determinada função baseada em seu valor de erro médio não normalizado $f\left(\mathbf{x}_{\text {best }}\right)-f\left(\mathbf{x}^{*}\right)$. Uma vez que $S R$ foi determinado para todos os algoritmos, o Score $_{2}$ é calculado como

$$
\text { Score }_{2}=\left(1-\frac{S R-S R_{\text {min }}}{S R}\right) \times 50
$$

onde $S R_{\text {min }}$ é o menor $S R$ entre todos os algoritmos. A pontuação final é a soma $S c o r e_{1}+$ $\mathrm{Score}_{2}$.

A Tabela 3 mostra a pontuação de cada esquema de mutação nos problemas adotados, enquanto que a Figura 1 mostra os boxplots dos erros, $f\left(\mathbf{x}_{\text {best }}\right)-f\left(\mathbf{x}^{*}\right)$, obtidos nas 30 execuções por cada esquema de mutação em cada um dos problemas. 


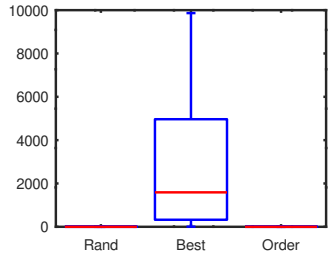

(a) Problema 1

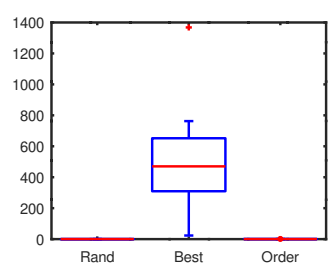

(e) Problema 5

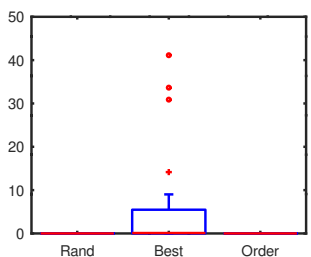

(i) Problema 9

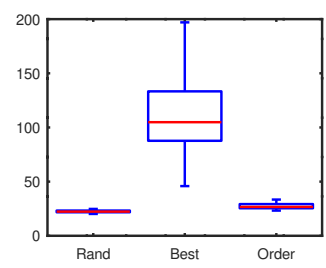

(m) Problema 3

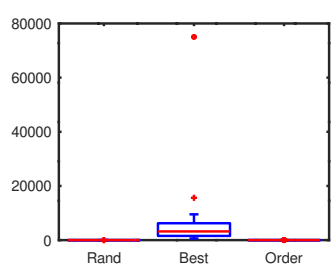

(q) Problema 7

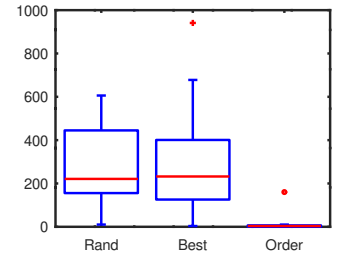

(b) Problema 2

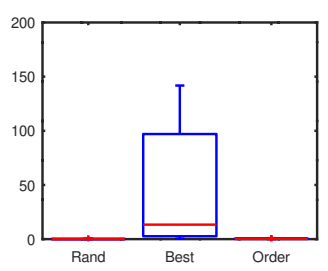

(f) Problema 6

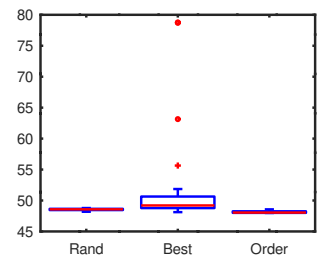

(j) Problema 10

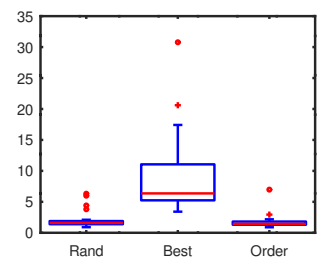

(n) Problema 4

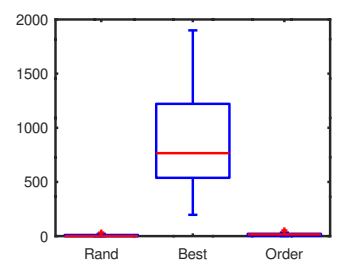

(r) Problema 8

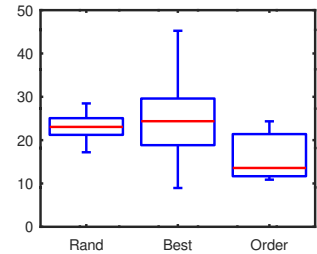

(c) Problema 3

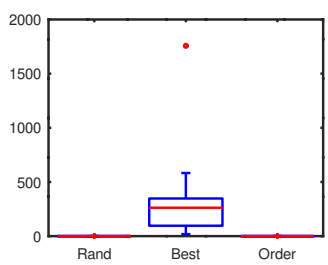

(g) Problema 7

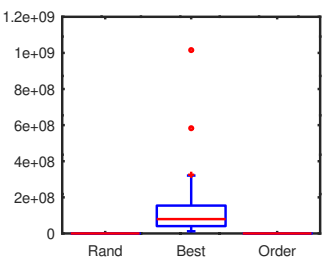

(k) Problema 1

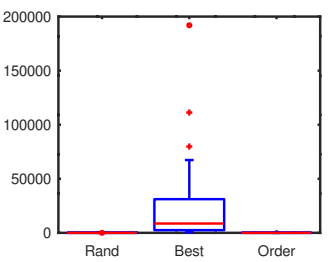

(o) Problema 5

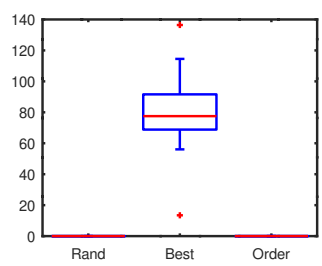

(s) Problema 9

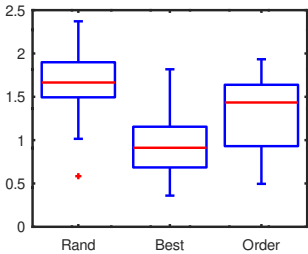

(d) Problema 4

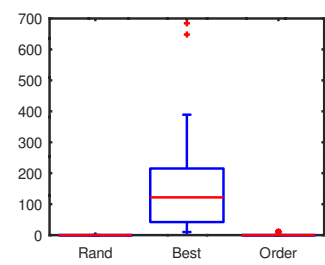

(h) Problema 8

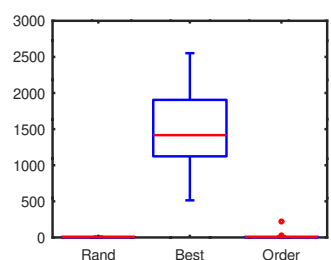

(1) Problema 2

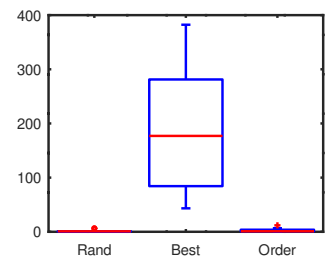

(p) Problema 6

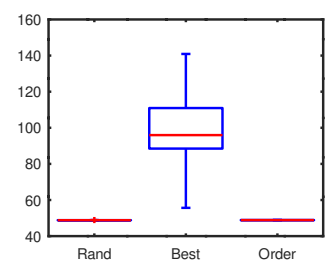

(t) Problema 10

Figura 1: Boxplots com os erros encontrados, $f\left(\mathbf{x}_{\text {best }}\right)-f\left(\mathbf{x}^{*}\right)$, nas 30 execuções de cada algoritmo em cada problema. As letras (a)-(j) referem-se à $D=10$ e as letras (k)-(t) referem-se à $D=20$. 
Tabela 3: Pontuação

\begin{tabular}{|c|c|c|c|}
\hline & DE/Rand & DE/Best & DE/Order \\
\hline Score $_{1}$ & 50 & 8 & 48.18 \\
\hline Score $_{2}$ & 50 & 25.47 & 42.19 \\
\hline Pontuação Final & $100\left(1^{\circ}\right.$ Lugar $)$ & $33.47\left(3^{\circ}\right.$ Lugar $)$ & $90.37\left(2^{\circ}\right.$ Lugar $)$ \\
\hline
\end{tabular}

\section{Discussão dos Resultados}

As pontuações apresentadas na Tabela 3 mostram que o esquema de mutação DE/Best foi o pior dentre os avaliados, com pontuação muito baixa quando comparado aos demais. Sua baixa eficiência também pode ser observada na Figura 1, cujos boxplots ficam majoritariamente acima dos outros. A principal razão deste desempenho ruim pode ser atribuída ao comportamento altamente elitista do DE/Best, contribuindo para convergências prematuras para ótimos locais. Uma vez preso nessas regiões, áreas promissoras do espaço de buscam deixam de ser visitadas e a falta de diversidade compromete o desempenho da abordagem para se desvencilhar e evoluir em busca da solução ótima.

Com relação aos esquemas DE/Order e DE/Rand, apesar dos resultados da Tabela 3 mostrarem o DE/Rand como o de melhor desempenho, o DE/Order ficou em $2^{o}$ Lugar e com pontuação próxima. O $p$-valor $(=0.000000853)$ obtido pela aplicação do teste não paramétrico de Kruskall Wallis [5] aos resultados, indica que há diferença estatística entre as abordagens. Para a comparação em pares, o também não paramétrico Wilcoxon Signed Test [6] foi aplicado. Os testes indicam diferença significativa entre as estratégias DE/Order e DE/Rand com a DE/Best, com $p$-valores iguais a 0.000003815 e 0.000009537 , respectivamente. Por outro lado, com $p$-valor $=0.277530251$, o teste não assegura a rejeição da hipótese nula na comparação entre DE/Order e DE/Rand, sugerindo que as distribuições entre estas duas abordagens são semelhantes. A semelhança dos desempenhos do DE/Order e DE/Rand pode ser visualmente percebida pelos boxplots da Figura 1, onde em praticamente todos os problemas eles possuem distribuições semelhantes.

Por fim, destaca-se aqui o DE/Order, pois além de se mostrar competitivo, principalmente quando comparado com o DE/Rand, ainda apresenta resultados substancialmente melhores em alguns problemas, como, por exemplo, os Problemas 2, 3 e 4 com $D=10$. O DE/Order, portanto, apresenta-se como uma alternativa: é competitivo ao DE/Rand, melhor em alguns problemas e também se beneficia de uma abordagem elitista semelhante ao DE/Best (Problema $4, D=10$ ), quando se propõe a ordenação, porém sem se estagnar em ótimos locais. Como exemplo, a Figura 2 ilustra a evolução das médias dos erros obtidos pelas abordagens no Problema 2, com $D=10$. Mesmo obtendo soluções melhores já no início da evolução, o DE/Best permanece nela por todo o restante da evolução. O DE/Order, por outro lado, evolui suas soluções na direção do ótimo, competindo bem com o DE/Rand.

\section{Conclusões}

Nesse trabalho avaliou-se o recententemente proposto esquema de mutação DE/Order em problemas de otimização monobjetivo com 10 e 20 dimensões. A performance desta estratégia foi comparada com outras duas, DE/Rand e DE/Best. Os resultados mostraram que o esquema DE/Best apresenta o pior desempenho, e a principal razão para isso recai sobre seu comportamento altamente elitista, apresentando convergência prematuramente para ótimos locais. Apesar do DE/Rand ter melhor desempenho que o DE/Order segundo uma métrica de scores, testes não paramétricos mostram que eles não possuem diferenças estatísticas. Destaca-se que o DE/Order se mostrou competitivo ao DE/Rand por não ter resultados muito piores que ele e ainda ter resulta- 


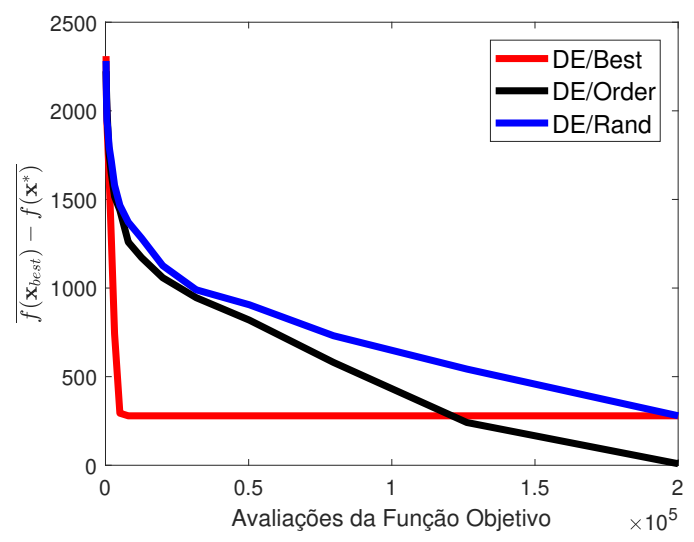

Figura 2: Evolução das médias dos erros, $f\left(\mathbf{x}_{\text {best }}\right)-f\left(\mathbf{x}^{*}\right)$, nas 30 execuções no Problema 2 com $D=10$.

dos significativamente melhores em alguns problemas, além de se beneficiar de estratégia elitista, como o DE/Best, porém sem ficar preso em ótimos locais. Trabalhos futuros poderão avaliar o $\mathrm{DE} /$ Order em problemas com restrições, além de formas híbridas e ensemble de variantes.

\section{Agradecimentos}

O terceiro autor agradece ao CNPq (306186/2017-9) e FAPEMIG (TEC PPM-174-18).

\section{Referências}

[1] Storn, R. e Price, K. Differential Evolution - A Simple and Efficient Heuristic for global Optimization over Continuous Spaces. Journal of Global Optimization, 11(4):341-359, 1997. DOI:10.1023/A:1008202821328.

[2] Bidgoli, A. A., Mahdavi, S., Rahnamayan, S. e Ebrahimpour-Komleh, H. GDE4: The Generalized Differential Evolution with Ordered Mutation. In Evolutionary Multi-Criterion Optimization. EMO 2019 - Deb K. et al. (eds). Lecture Notes in Computer Science, Springer, Cham. volume 11411, pages 101-113, 2019. DOI: 10.1007/978-3-030-12598-1_9.

[3] Kukkonen, S. e Lampinen, J. GDE3: the third evolution step of generalized differential evolution, Proceedings of the IEEE Congress on Evolutionary Computation. CEC 2005, Edinburgh, Scotland, volume 1, pages 443-450, 2005. DOI: 10.1109/CEC.2005.1554717.

[4] Wagdy, A., Hadi, A. A., Mohamed, A. K., Agrawal, P., Kumar, A e Suganthan, P. N.. Problem Definitions and Evaluation Criteria for the CEC 2021 Special Session and Competition on Single Objective Bound Constrained Numerical Optimization, Technical Report, Nanyang Technological University, Singapore.

[5] Kruskal, W. H., \& Wallis, W. A. Use of ranks in one-criterion variance analysis. Journal of the American statistical Association, 47(260), 583-621, 1952.

[6] Pratt, J. W. Remarks on zeros and ties in the Wilcoxon signed rank procedures. Journal of the American Statistical Association, 54(287), 655-667, 1959. 